
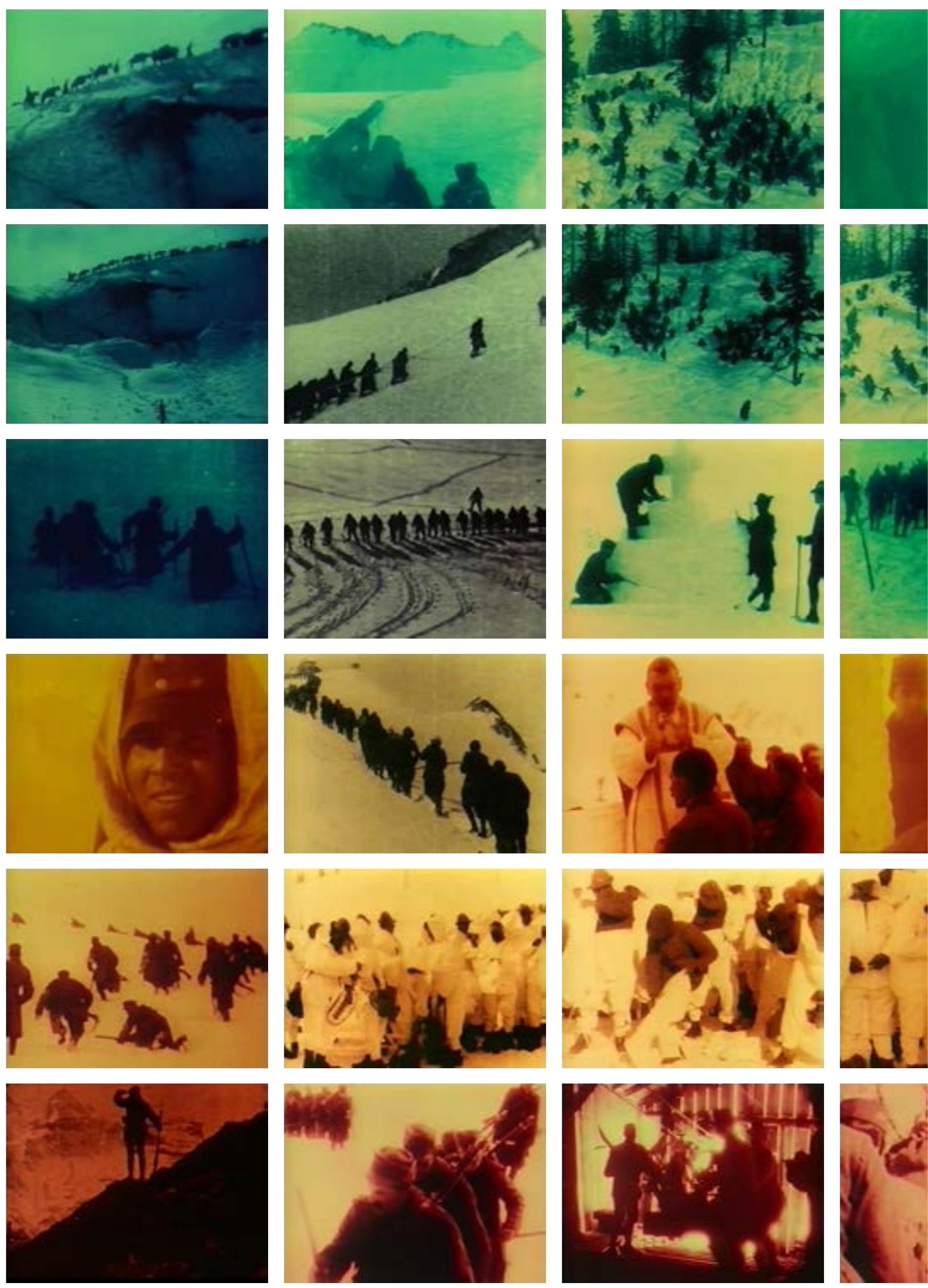


\title{
Riusi radicali. Soldati, sirene, deflagrazioni
}

\author{
Radical reuse. Soldiers, mermaids, deflagrations
}

\begin{abstract}
Reuse is a process that deals not only with objects, architectures and territories, but also with images and collective perception. It goes beyond the physical reality and produces a space having less delimited meanings, more difficult to be defined because of its immateriality, although not less necessary.

Montage, giving new meanings to images from archives, has been since long an artistic methodology used for movies, photography, visual arts. In this context, some artworks, like the most interesting architectonic reuses, do not re-propose slavishly pre-existing realities. On the contrary, thorough a process of estrangement and montage, they relaunch, open to unexpected outcomes.

This text proposes an analysis of some works straddling the beginning of the millennium, by Yervant Gianikian, Angela Ricci Lucchi and Joan Fontcuberta. These authors, by different means, suggest the existence of a common ground, a possible map of the contemporary artistic production dealing with alpine territories. Nitrate ghosts of living beings suspended in a nearly abstract white, digital landscape, mermaids' skeletons. Images differ greatly from one another and displace the observer. By deconstructing the mechanisms behind communications produced by different systems of power (scientific, political, etc.), they dismantle prejudices and established visions. They do not oppose new realities to existing ones, but with the irruption of the unexpected, of discrepancy and heterogeneity, compared to conventions and to the foreseeable, they activate the observer, bounced out of the comfort zone of the passive spectator.
\end{abstract}

\section{Laura Cantarella}

(Savigliano, 1972) is a photographer, independent researcher, and lecturer. She alternates her activities as an author with those of planning and coordinating territorial investigations, with particular attention given to fragile territories.

She earned a degree in Architecture and a $\mathrm{PhD}$ in Humanities. Her works have been exhibited in numerous museums and cultural institutions. She is one of the winners of Terna Award for Contemporary Art and one of the finalists of Francesco Fabbri Award.

\begin{abstract}
Alberto Momo
(Turin, 1972), filmmaker and director of architectural documentaries, has presented his films at the major international festivals, including Venice, Locarno, Rotterdam, and Montreal. He and Donatello Fumarola founded the production company Zomia and published the book of conversations Atlante sentimentale del cinema. He has collaborated with Rai 3 and Rai 4, and curated the film section of the 2014 Architectural Biennale, with a large installation at the Arsenale. His film Eliorama won best Italian documentary at the 2006 Torino Film Festival.
\end{abstract}

Keywords

Reuse, visual art, photography, image, imaginary. 
Il riuso è un procedimento che non riguarda solo gli oggetti, i manufatti architettonici e i territori, ma anche le immagini e gli immaginari. Va dunque oltre la realtà fisica, e produce uno spazio di significati meno delimitabile, di più difficile definizione perché immateriale, ma non per questo meno necessario.

Il montaggio e la risemantizzazione di immagini di archivio costituiscono da tempo dispositivi utilizzati dal cinema, come dalla fotografia e dalle arti visive in generale. In questo contesto esistono alcuni lavori che operano, così come i più interessanti riusi architettonici, non riproponendo pedissequamente realtà preesistenti, ma, attraverso un procedimento di straniamento e montaggio, rilanciando, aprendo a esiti inaspettati.

In questo testo si propone una lettura di alcuni lavori, che si situano a cavallo del millennio, di Yervant Gianikian, Angela Ricci Lucchi e Joan Fontcuberta, autori che, con modalità diverse, suggeriscono l'esistenza di un terreno comune, una mappa possibile per la produzione contemporanea che si occupa dei territori alpini.

\section{Su tutte le vette è pace}

Immagini ritrovate, ripulite e restaurate, rifilmate e a volte ricolorate. Rallentate, reinquadrate e rimontate. Il processo di riuso operato dai due cineasti italiani Yervant Gianikian e Angela Ricci Lucchi sulle immagini d'archivio, sui fotogrammi della Storia, appare come un intervento radicale di risignificazione del reale, della nostra memoria e dei nostri immaginari.

Pensiamo in particolare a un film della fine degli anni Novanta, Su tutte le vette è pace (1998) che lavora sulle immagini d'archivio della Guerra Bianca: la documentazione filmata dei fronti di guerra, italiano e austro-ungarico, sulle Alpi Orientali durante la prima guerra mondiale. Parte dei materiali provengono da quell'archivio Comerio, ritrovato e acquisito da Gianikian e Ricci Lucchi, che ha rappresentato l'oggetto di studio sullo sguardo coloniale nel precedente Dal Polo all'equatore (1986). Altro proviene da nuove ricerche presso il Film Archive di Vienna, come presso istituzioni ungheresi, tedesche, francesi e americane.
Su tutte le vette è pace, da un verso del Canto della notte di un viandante di Goethe, si apre proprio sull'immagine del wanderer, con una figura umana stagliata in controluce sul profilo della montagna annegata in una ricoloritura rossa, come la sequenza successiva di esplosioni rosse sulla neve. E subito ci mette di fronte alle contraddizioni dei nostri immaginari, e del nostro godimento estetico. Alla violenza latente di quel sublime che molto spesso informa la rappresentazione della montagna e delle Alpi in particolare.

Contraddizioni che sono rinforzate dalle sequenze successive. File di uomini come cordate impegnate in un'ascesa. La ripresa di una teleferica dove uomini raggiungono le vertiginose altezze imbracciando un paio di sci. Potrebbero essere le immagini di una spedizione, o della domesticazione della montagna a fini ludici. Ma qui la conquista dell'altezza, delle vette, ha un altro significato. Per l'esercito austro-ungarico questa ascesa rappresenta la conquista dei punti strategicamente rilevanti: come sappiamo bene i luoghi che oggi riteniamo panoramici, i luoghi della contemplazione estetica, sono gli stessi punti privilegiati dell'osservazione militare. La nostra esperienza di godimento dello spazio nasconde dentro di sé un'illusione di dominio, di conquista.

Alternando le immagini dei due fronti, sugli opposti versanti delle Alpi, il film di Gianikian e Ricci Lucchi ci interroga poi su un altro carattere invisibile, che costituisce però lo stesso centro simbolico della guerra: la linea di confine. Una linea mai così mobile e impercettibile come sulle cime ghiacciate delle Alpi, dove questa linea, in modo apparentemente invisibile, si sposta e si riconfigura senza soluzione di continuità.

L'azione dei due cineasti mette così lo spettatore di fronte all'enigma delle immagini e della rappresentazione, obbligandolo a riconsiderare la natura profonda, verrebbe da dire ontologica, dei propri immaginari. Ma Su tutte le vette è pace non è solo questo. Come si racconta la Storia? Quali retoriche sottendono l'autorappresentazione del racconto storico, e in particolare della guerra? Masse anonime, celebrazione della potenza, la sparizione dell'individuo. $\mathrm{Ci}$ sono sequenze assolutamente sorpren- 
denti nel film. Soldati italiani impegnati a scolpire un altare e una croce nei ghiacci. Altri soldati filmati mentre indossano tute mimetiche bianche come in un film di fantascienza. Tutto sembra portare a un'astrazione, fino a quando vediamo i corpi cadere, falciati dalle linee di fuoco, nella neve. La vita, la morte, la fatica. La Camera Analitica di Gianikian e Ricci Lucchi attraverso rallentamenti e re-inquadrature si sforza a rivelare volti, gesti e azioni del soldato anonimo. Anche se la sequenza più memorabile ha come protagonista un animale, un mulo, altro eroe invisibile della guerra combattuta sulle Alpi. Nel finale del film lo vediamo arrancare nella neve, sprofondare sotto il peso dei materiali, spinto e tirato dai soldati fino allo sfinimento, mentre la voce del coro di Giovanna Marini intona quello che ora ci sembra un requiem.

\section{Orogenesis}

Apparentemente una serie di spettacolari paesaggi montani privi di qualunque presenza umana, adatti ad essere ospitati in un libro patinato coffee table, Orogenesis (2002) è invece un progetto artistico sulla rappresentazione dello spazio, il cui titolo si riferisce a quella parte della geografia fisica che si occupa della formazione delle montagne.

L'autore, Joan Fontcuberta, è un artista, curatore e docente catalano, la cui ricerca sullo statuto iconico della fotografia indaga il modo in cui le persone fanno esperienza delle immagini e in generale di ciò che hanno di fronte agli occhi. Cresciuto nell'agenzia pubblicitaria del padre, durante la dittatura e la censura franchista, il suo percorso muove dalle scienze dell'informazione e si snoda nell'arte contemporanea, esplorando spazi di tangenza tra scienza, potere e rappresentazione.

In Orogenesis Fontcuberta adotta come dispositivo di produzione delle immagini un software, realizzato in origine a scopo militare, in grado di tradurre informazioni cartografiche in paesaggi virtuali tridimensionali che, nonostante l'apparenza fotografica, non hanno un referente esterno catturato attraverso un dispositivo ottico.

Fontcuberta opera un'azione sovversiva nei confronti della rappresentazione di radice surrealista, utilizzando la tecnologia contemporanea: invece di mappe, fornisce al software immagini di paesaggio esistenti, come ad esempio pitture di Friedrich, Dali, Turner, Hokusai, Courbet e fotografie di Legret, Atget, Watkins.

Il software, diligentemente, esegue e produce autonomamente paesaggi. Il progetto pubblicato da Aperture nel 2005 con il titolo di Landscapes without memory, dichiara l'inganno e la natura enigmatica dell'immagine, affiancando le due immagini: quella fornita al software dall'autore e quella prodotta dallo stesso.
La tecnologia utilizzata tradisce la datazione del progetto artistico, ma le questioni che pone circa la formazione di immagini e immaginari si presentano come assolutamente contemporanee. Se i paesaggi di Orogenesis, richiamano il labirinto iconico dell'industria dell'intrattenimento digitale, ponendo al centro lo spettacolo e la spettacolarizzazione, il software utilizzato da Fontcuberta può essere letto anche come l'apparato visivo umano che, incapace di uno sguardo innocente sul reale, costruisce la visione sulla base di immagini previe, con tutti i rischi annessi, se nutrito a base di immagini di consumo e stereotipi.

Orogenesis suggerisce inoltre come ogni rappresentazione della natura sia costruzione culturale, ideologica, economica e politica, e come non abbia origine dalla natura stessa, ma da altre immagini. Sarebbe cioè rielaborazione, risultato di un riuso. Fontcuberta pone la questione della verosimiglianza fotografica, ma anche dei limiti della costruzione dell'immaginario, della necessità di decostruirlo costantemente. Effetto collaterale della diminuita fiducia nell'immagine come mezzo di duplicazione del reale è l'aumento del suo potenziale espressivo, artistico e progettuale.

\section{Hydropitecus}

«All'inizio degli anni '50, il geologo e sacerdote gesuita Jean Fontana, originario dell'Alta Provenza, ha trovato degli scheletri pietrificati corrispondenti agli specimen chiamati con il nome scientifico di Hydropitecus, la cui morfologia assomiglia molto alle sirene della mitologia. Questa stupefacente scoperta paleontologica sconvolge tutta la teoria dell'Evoluzione! Fontana prosegue le sue ricerche trovando altri fossili di idropiteco».

Fontcuberta introduce con queste parole Les hydropithèques de Digne (2000-2011), un progetto realizzato su invito del CAIRN centre d'art, istituzione che ha come obiettivo interpretare le aree rurali delle Alpi provenzali attraverso residenze artistiche. In questo modo negli ultimi 20 anni, ha prodotto numerose opere di arte contemporanea in aree pubbliche, distribuite sul territorio del Géoparco dell'Alta Provenza che comprende svariati comuni su più di 200.000 ettari, riconosciuto oggi come patrimonio dell'UNESCO.

Fontcuberta realizza un'opera permanente e site specific innestata nella roccia (senza precedenti nella produzione dell'autore) nella Riserva Geologica di Digne: uno scheletro di una creatura mezza uomo e mezzo pesce. A questa affianca una finzione narrativa composta da una rivista scientifica, una serie di immagini fotografiche e alcuni video che documentano il ritrovamento degli scheletri di Hydropitecus. Intesse la storia di finzione con dati reali, come ad esempio la figura di Albert-Félix de 
Lapparent, sacerdote, geologo e paleontologo, di cui Fontana (impersonificato nelle fotografie dallo stesso Fontcuberta) sarebbe stato allievo.

Come sottolinea la curatrice Nadine Gomez-Passamar il progetto «è una strategia retorica che permette all'artista di immergersi nei discorsi sull'autorità, nei discorsi della scienza, della religione, della politica. In questo caso si tratta al tempo stesso di scienza (tramite la riserva naturale che allestisce siti) e di museo (che posizionando delle opere all'aperto museifica la natura), i quali funzionano come luogo di costruzione dei saperi e di legittimazione dei valori».

Con uno stile (ma non con un obiettivo) documentaristico, Fontcuberta lavora instillando il dubbio nell'osservatore. L'intento è quello di attivare la reazione critica dello spettatore, la sua capacità di mettere in dubbio non soltanto l'esistenza dell' $\mathrm{Hi}$ dropitecus, ma di tutto ciò che viene somministrato come verosimile da qualunque autorità, anche quella scientifica. Si tratta di un movimento allo stesso tempo lieve e radicale nei confronti delle immagini veicolate da qualunque tipo di sistema comunicativo. Anche in questo caso la cosiddetta veridicità dell'immagine fotografica viene smascherata come pura convenzione.

Fontcuberta procede con precisione (nella realizzazione e della documentazione) e allo stesso tempo in modo ludico, suggerendo le enormi possibilità che si aprono in campo artistico e culturale dall'uso consapevole dell'ironia sovversiva, ampiamente sottoutilizzata.

Nel sito di recensioni per turisti Tripadvisor due visitatori citano scheletri fossili di sirenidi e uno spettacolare scheletro fossile di sirenide (se recependo la finzione come reale o accettando il gioco proposto dall'autore, non è dato sapere), a conferma del fat-

Joan Fontcu

La tanières des hydropithèques, Grotte de Barles, 2011 (c) Musée Gassendi.

Fig. 2 Joan Fontcuberta,

La falaise des amoureux, abris sous roche du Bès, 2011 C Musée Gassendi.

Fig. 3

Joan Fontcuberta, Hydripithèques, Scène de crime, Squelette de Beaujeu, 2011 (C) Musée Gassendi. to che il lavoro di Fontcuberta è più che mai utile e necessario contro ogni forma di certificazione della realtà (ivi compresa ogni tematizzazione di luoghi) che relega il visitatore, il viaggiatore, l'essere umano nel ruolo di spettatore passivo, di consumatore.

Fontcuberta costruisce la finzione a partire dal riuso di elementi reali, (un luogo, un nome, un evento) e opera una decostruzione del linguaggio della comunicazione associata al potere, sia esso politico, religioso, scientifico, mediatico, svelandone meccanismi di funzionamento e seduzione. Apre così ampi spazi di possibilità per l'osservatore che, se consapevole delle regole del gioco, non può restare in una posizione di recettore, ma è spontaneamente chiamato a una posizione proattiva, una posizione che molto ha a che fare con il viaggiatore o il progettista che, partendo da un punto nello spazio e nel tempo, cercano il rilancio, il passo oltre.

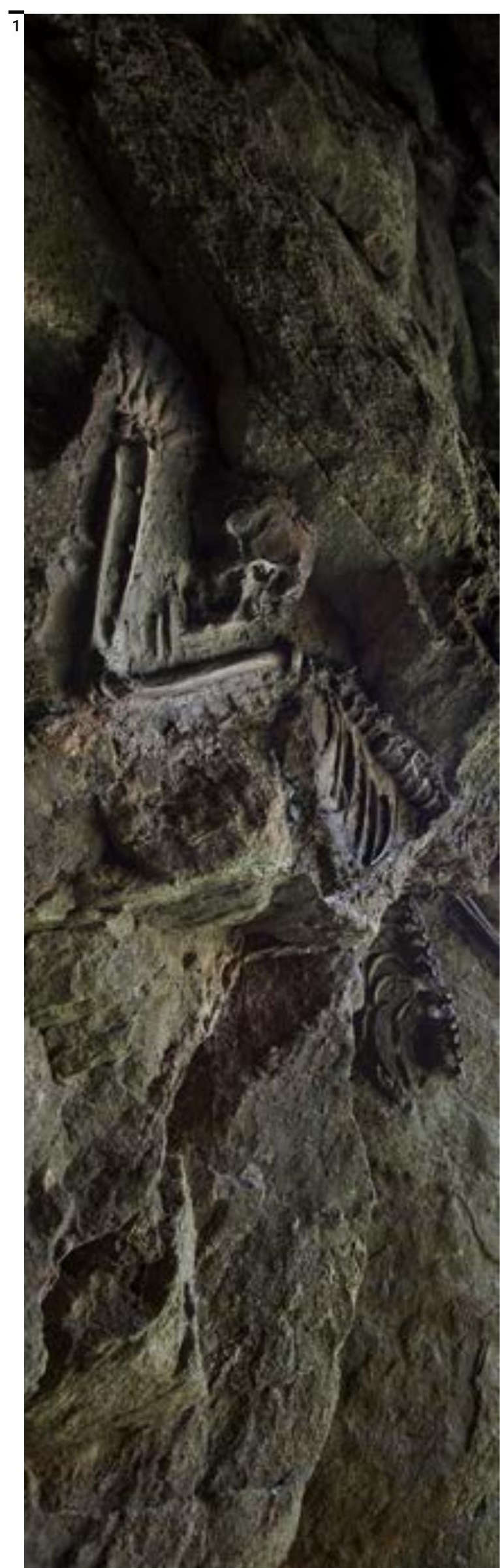



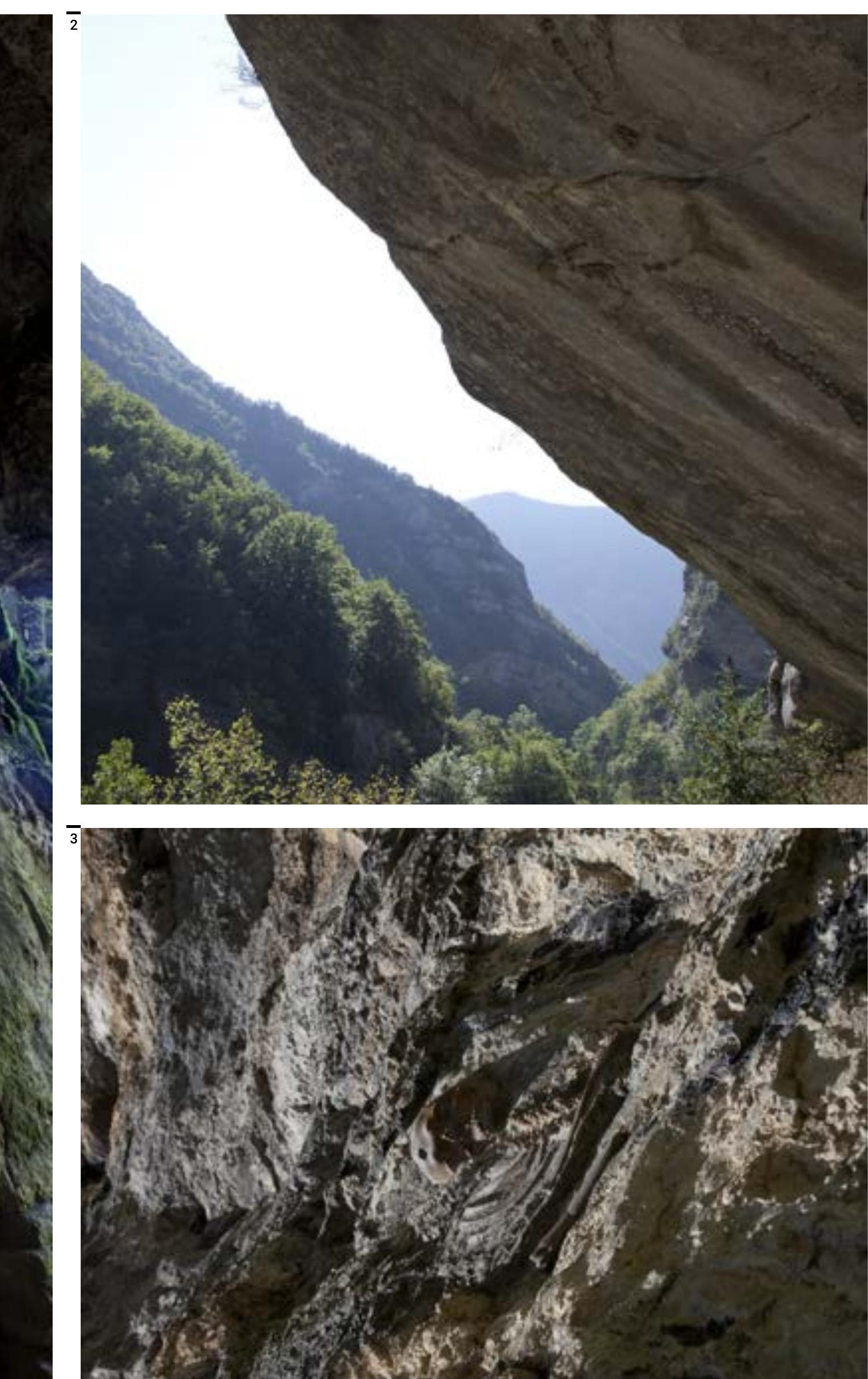
Fantasmi in nitrato di esseri viventi immersi in uno spazio bianco quasi astratto, paesaggi digitali, scheletri di sirene. Immagini molto diverse che spiazzano l'osservatore e che, decostruendo i meccanismi della comunicazione prodotta da diversi sistemi di potere, fanno deflagrare pregiudizi e visioni precostituite. Non oppongono verità altre a verità pre-esistenti, ma con l'irruzione dell'inatteso, del discordante e dell'eterogeneo rispetto alla norma e al prevedibile, attivano lo sguardo dell'osservatore, sbalzato fuori dalla comfort zone dello spettatore passivo.

Il riuso radicale di immagini e immaginari, così come adottato dagli autori cui si è rivolto questo testo, si dà innanzitutto nella ricerca e nella selezione dei materiali esistenti, quindi necessariamente anche in una sottrazione. In secondo luogo si produce in una reinvenzione dei materiali - che implica un'idea del riuso che recupera una pre-esistenza, ma rilancia attivamente in avanti, avendo ben a mente il presente - operando uno straniamento che contiene in sé un potenziale liberatorio e dinamico per l'osservatore: il grado zero del progetto.

Il riuso efficace degli immaginari, in un contemporaneo sovrabbondante di immagini, difficilmente potrà prescindere dagli elementi messi in gioco da questi autori: sottrazione, reinvenzione, straniamento. Come risultato auspicabile, il tentativo di disinnescare ogni facile spettacolarizzazione e tematizzazione. 


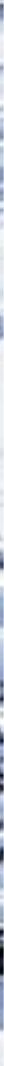

